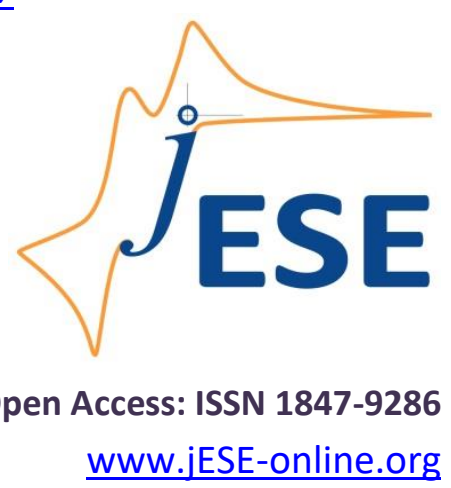

Original scientific paper

\title{
Numerical modelling of buried pipelines under DC stray current corrosion
}

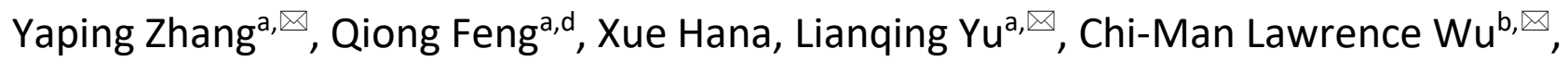 \\ Siu-Pang $\mathrm{Ng}^{\mathrm{b}}$, Xiao Tang \\ ${ }^{a}$ College of Science, China University of Petroleum (East China), Qingdao, 266580, P. R. China \\ ${ }^{b}$ Department of Materials Science and Engineering, City University of Hong Kong, Hong Kong, SAR, \\ P.R. China \\ ${ }^{c}$ College of Mechanical and Electrical Engineering, China University of Petroleum (East China), \\ Qingdao, 266580, P. R. China \\ 'Semiconductor Lighting Technology Research and Development Center, Institute of \\ Semiconductors, Chinese Academy of Sciences, Beijing, 100083, P. R. China
}

\Corresponding authors: E-mail zhangyp@upc.edu.cn; ivy2000@163.com; lawrence.wu@cityu.edu.hk

Received: July 7, 2018; Revised: October 29, 2018; Accepted: November 5, 2018

\begin{abstract}
Corrosion of buried pipelines caused by stray currents is becoming a serious industrial and environmental problem. It is therefore necessary to study corrosion mechanisms of buried pipelines under DC stray currents in order to propose effective anti-corrosion measures. Since measurement of the potential is one of important ways to identify stray current intensity, the COMSOL Multiphysics software was used to simulate stray current corrosion dynamics of buried pipelines. It was also used to calculate the distribution and intensity changes of electrolyte potential in the cathodic protected system by solving Laplace's threedimensional equation. The obtained results showed that increased applied voltage leads to more positive shift of a pipeline potential, resulting in acceleration of stray current corrosion. On the contrary, increased soil resistivity can retard the corrosion process. The protected pipeline with a sacrificial anode suffers less corrosion interference than unprotected pipeline. Two crossed arrangement of pipelines makes no difference in corrosion of protected pipeline, but affects greatly on unprotected pipeline.
\end{abstract}

\section{Keywords}

Stray current corrosion; numerical modeling; buried pipelines

\section{Introduction}

Stray currents arising from railway systems can induce corrosion of buried pipeline structures and result in severe damage [1]. Basically, there are four types of corrosion [2], involving general 
corrosion (chemical in nature), concentration cell corrosion (caused by differences in the electrolyte concentration), galvanic corrosion (caused by different metals), and stray current corrosion (caused by external electrical sources). Among all corrosion types, just the stray current corrosion (SCC) is considered as the most serious one. SCC is caused by the flow of stray currents through pipelines, which usually occurs on their external surfaces. The consequences of SCC have been manifested as severe localized pitting and pin holes formed on metal surfaces at the place where stray currents leave the pipeline surface $[3,4]$.

A routine way to mitigate the SCC of pipelines is to install sacrificial anodes or apply a current to inappropriate bedding for protected structures. Both sacrificial anodes and applied current belong to the cathodic protection procedure. Cathodic protection is benefit for charges transport from the metal pipeline to the anode [5]. Corrosion easily happens because part of a pipeline in the soil is anodic and the other part in the air is cathodic. Therefore, the goal of cathodic protection is to apply a direct current to the steel and provide a sacrificial anode [6]. With the development of technology, the numerical modeling becomes very convenient and accurate method for dealing with SCC problems. There are three kinds of numerical analysis for cathodic protection systems, including finite difference method, boundary element method and finitude method. Among these methods, the boundary element is suited to off-shore structures as a method capable to infer the distribution of potential and current densities along the metallic structure/electrolyte interface [7]. At the other side, the finitude method is well suited to cathodic protected pipelines.

COMSOL Multiphysics is the simulation software based on the finitude method for corrosion numerical calculations. In this paper, the COMSOL Multiphysics software is used to evaluate the effect of stray current on the protected/unprotected pipelines resulting from a nearby cathodic protection system.

\section{Theory}

\section{Governing equation}

The boundary and initial conditions were set during the simulation, of the protected pipeline and anode ball constituting a whole [8]. The anode ball acts as an anode and produces the anodic oxidation reaction which defines the anode boundary condition. The protected pipeline acts as a cathode for oxygen reduction which defines the cathode boundary condition. The unprotected pipeline can self-corrode in the soil environment, where anode and cathode reactions occur simultaneously on the pipeline surface. The electrode boundary conditions should be set in two ways. The initial value of electrolyte potential is set to $-0.90 \mathrm{~V}$, and the electrolyte potential is set zero at infinity.

The principles of cathodic protection of underground structures have already been discussed in detail [9]. The soil is treated as a homogeneous medium with a uniform conductivity. The potential ( $\phi$ )distribution is governed by the Laplace's equation

$\nabla^{2} \phi=0$

and the current density $(i)$ is related to the electric field by the Ohm's law as follows

$i=-\kappa \nabla \phi$

In eq. (2), $\kappa$ is electrical conductivity of the soil.

The boundary conditions are defined as

$\partial \phi / \partial m=0$ 
where $m$ is the number of electrons transferred directly. Eq. (3) is defined for the region between the insulation layer of unprotected pipeline without any defect and the soil-air surface.

$$
\begin{aligned}
& i_{\mathrm{a}}=\exp \left(\eta_{\mathrm{a}} / \beta_{\mathrm{a}}\right) \\
& i_{\mathrm{c}}=i_{0, \mathrm{c}} \exp \left(-\eta_{\mathrm{c}} / \beta_{\mathrm{c}}\right)
\end{aligned}
$$

In boundary conditions defined by eqs. (4) and (5), $i_{a}, i_{c}, i_{0, a}, i_{0, c}$ are anodic and cathodic current and exchange current densities, while $\beta_{\mathrm{a}}, \beta_{\mathrm{c}}$ and $\eta_{\mathrm{a}}, \eta_{\mathrm{c}}$ are corresponding Tafel slopes and surface overpotentials, respectively. The boundary conditions defined by eqs. (4) and (5) represent the electrode surface kinetics which strictly follows the Tafel kinetics.

\section{Reaction chemistry}

Three kinds of electrochemical reactions happen on the steel interface, including iron oxidation, oxygen reduction, and hydrogen evolution [10].

(1) Hydrogen evolution reaction

Anode reaction: $2 \mathrm{Fe} \rightarrow 2 \mathrm{Fe}^{2+}+4 \mathrm{e}^{-}$

Cathode reaction: $4 \mathrm{H}^{-}+4 \mathrm{e}^{-} \rightarrow 4 \mathrm{H}_{2}$

$$
4 \mathrm{H}_{2} \mathrm{O}+4 \mathrm{e}^{-} \rightarrow 2 \mathrm{H}_{2}+4 \mathrm{OH}^{-}
$$

(2) Oxygen reduction reaction

Anode reaction: $2 \mathrm{Fe} \rightarrow 2 \mathrm{Fe}^{2+}+4 \mathrm{e}^{-}$

Cathode reaction: $\mathrm{O}_{2}+4 \mathrm{H}^{+}+4 \mathrm{e}^{-} \rightarrow 2 \mathrm{H}_{2} \mathrm{O}$

$$
\mathrm{O}_{2}+2 \mathrm{H}_{2} \mathrm{O}+4 \mathrm{e}^{-} \rightarrow 4 \mathrm{OH}^{-}
$$

When stray currents induce pipeline corrosion, the Faraday' law should be conformed [11]

$$
K_{\mathrm{c}}=\frac{J_{\mathrm{n}} M}{n F}
$$

In eq. (6) $K_{\mathrm{c}}$ is the amount of metal corrosion, $J_{\mathrm{n}}$ is the normal current density, $M$ is atomic mass of the metal, $F$ is the Faraday' constant, and $n$ is the number of electrons required for the reaction. For a given metal and reaction, parameters $M, F$, and $n$ are constant, and so $K_{\mathrm{c}} \infty J_{\mathrm{n}}$

\section{Modeling Configuration}

In order to ensure the accuracy of simulation and save computing resources, according to the length and diameter of actual pipelines, we setup a 1:100 scale model to build up the simulation model (schematically shown in Figure 1). The length, width and height of the scale model were set as $500,800,500 \mathrm{~mm}$, respectively, and the length of pipelines was set proportional to its diameter. The distance of sacrificial anode to the pipeline was set proportional to the actual project. The pipelines are evenly coated with epoxy resin. Epoxy resin coating on pipeline surface should be strictly in accordance with SHT3022-2011 code for corrosion prevention design of petrochemical equipment and pipeline coatings.

Models were built up to simulate the corrosion of the protected/unprotected structure due to stray currents. Two study cases with different arrangements of pipelines were investigated, involving parallel and crossed arrangements of protected and unprotected pipelines under different interference conditions. Geometrical, physical and electrochemical model parameters are listed in Tables 1 and 2. A tank was filled with the soil having measured electrical resistivity of $50.0 \Omega \cdot m$, which is consistent with the reference [12]. The protected cathode and unprotected structure were placed in the tank at a height of $20 \mathrm{~cm}$ from the bottom. Two break points were set at both ends of the pipeline. The copper plates were used to insure the homogeneous potential differences, and a constant direct voltage was applied to the copper plates. The solution side potential of the 
protected/unprotected structure was scanned with a saturated copper sulfate reference electrode (CSE). Stray current was picked upon the unprotected pipeline and the protected pipeline was connected with a sacrificial anode. Physical fields are added to the geometric model, and the secondary current distribution model (corrosion process satisfy the Ohm's law and activation loss during charge transfer) in the corrosion module is selected. The anode and cathode reactions in the corrosion process satisfied the dilute material transfer model in the electrochemical module.

As shown in Figure 2, the sacrificial anode has more positive potential, and so, electric field lines are emitted from it and terminate at the cathodically polarized structure having more negative potential. The current in the structure flows towards the anode, and regions where currents enter will be at a more negative potential, protecting thus the structure from corrosion. Sacrificial anode protection is equivalent to a direct current flowing to the pipeline compulsively, resulting in negative potential shift and reducing the corrosion rate of the structure.
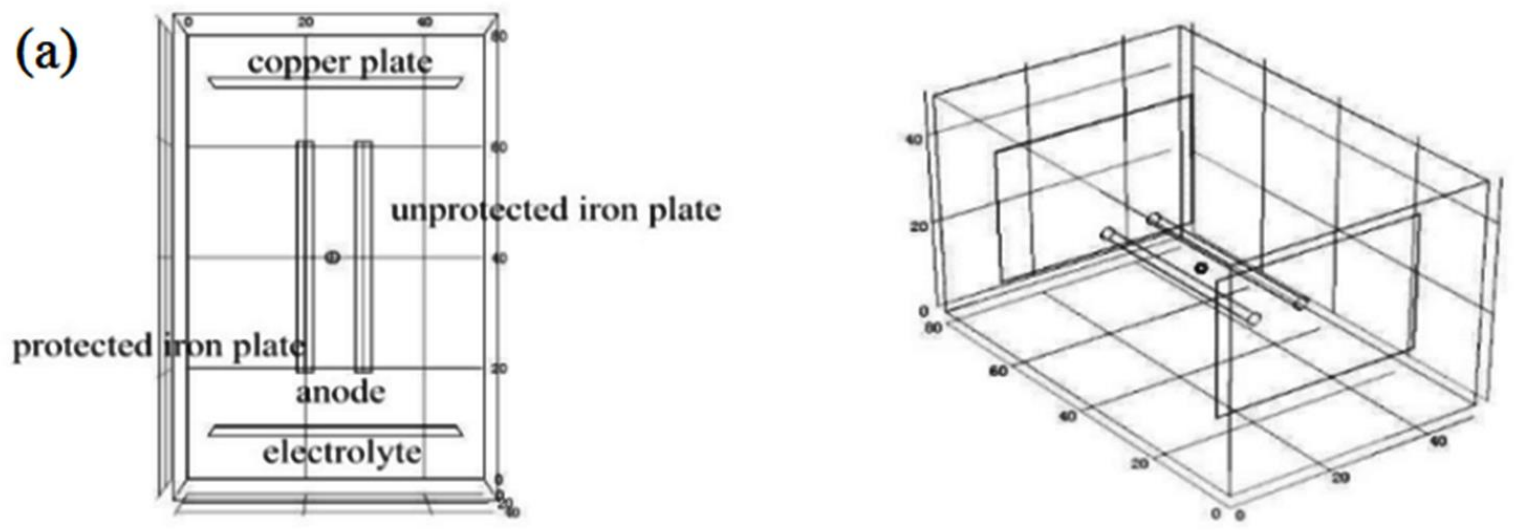

(b)
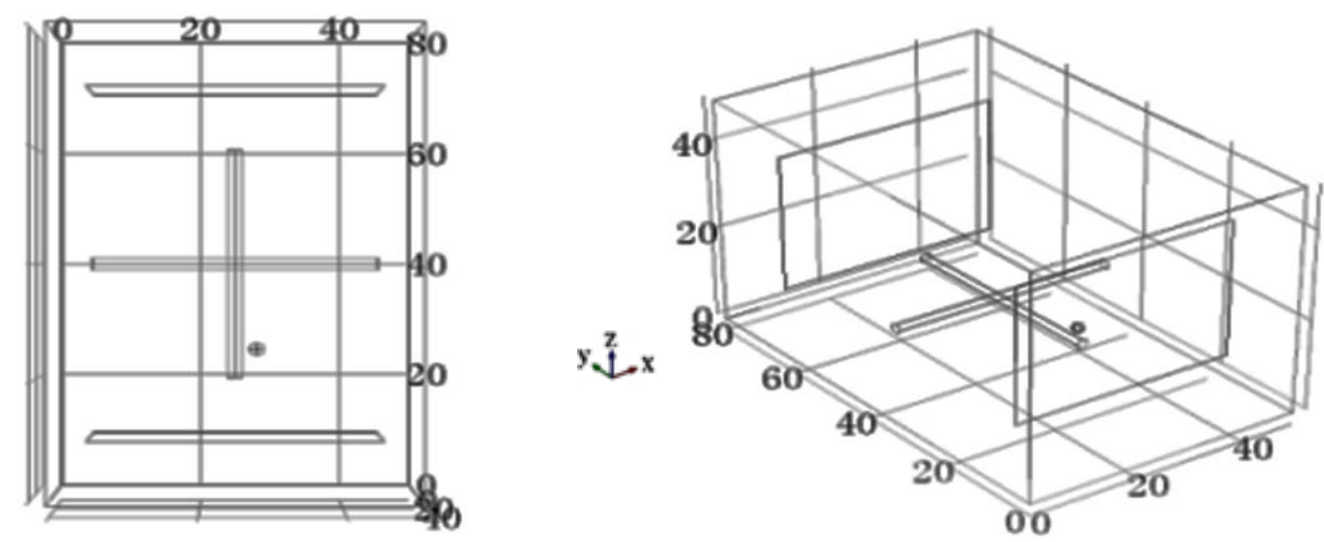

Fig. 1. Schematic view of models: (a) parallel pipelines; (b) crossed pipelines

Table 1. Specification of physical model parameters

\begin{tabular}{ll}
\hline Quantity & Value \\
\hline Protected pipeline length & $400 \mathrm{~mm}$ \\
Unprotected pipeline length & $400 \mathrm{~mm}$ \\
Protected pipeline diameter & $4 \mathrm{~mm}$ \\
Unprotected pipeline diameter & $4 \mathrm{~mm}$ \\
Anode diameter & $2 \mathrm{~mm}$ \\
Soil resistivity (if not specified) & $50.0 \Omega \cdot \mathrm{m}$ \\
Anode current density (if not specified) & $100 \mathrm{~A} / \mathrm{m}^{2}$ \\
\hline
\end{tabular}


Table 2. Electrochemical model parameters

\begin{tabular}{|c|c|c|}
\hline Parameter name & Value & Description \\
\hline$F$ & $96485 \mathrm{C} / \mathrm{mol}$ & Faraday constant \\
\hline$E_{\text {eq_Fe }}$ & $-0.76 \mathrm{~V}$ & Equilibrium potential of $\mathrm{Fe}-\mathrm{Fe}^{2+}$ \\
\hline$i_{0 \_} \mathrm{Fe}$ & $7.1 \times 10^{-5} \mathrm{~A} / \mathrm{m}^{2}$ & Exchange current density of $\mathrm{Fe}-\mathrm{Fe}^{2+}$ \\
\hline$\beta_{\mathrm{Fe}}$ & $0.41 \mathrm{mV} /$ decade & Ion Reduction Tafel Slope \\
\hline$E_{\text {eq_ } \mathrm{H}_{2} \mathrm{O}}$ & $-1.03 \vee$ & Equilibrium potential of $\mathrm{H}_{2} \mathrm{O}-\mathrm{H}_{2}$ \\
\hline$i_{0 \_} \mathrm{H} 2 \mathrm{O}$ & $0.11 \mathrm{~A} / \mathrm{m}^{2}$ & Exchange current density of $\mathrm{H}_{2} \mathrm{O}-\mathrm{H}_{2}$ \\
\hline$\beta_{\mathrm{H} 2 \mathrm{O}}$ & $0.15 \mathrm{mV} /$ decade & $\mathrm{H}_{2} \mathrm{O}$ reduction Tafel slope \\
\hline $\mathrm{E}_{\text {eq_O} 2}$ & $0.189 \mathrm{~V}$ & Equilibrium potential of $\mathrm{O}_{2}$ and $\mathrm{OH}^{-}$ \\
\hline$i_{0 \_} \mathrm{O}_{2}$ & $7.7 \times 10^{-7} \mathrm{~A} / \mathrm{m}^{2}$ & Exchange current density of $\mathrm{O}_{2}$ and $\mathrm{OH}^{-}$ \\
\hline$\beta_{\mathrm{O}_{2}}$ & $-0.18 \mathrm{mV} /$ decade & Oxygen reduction Tafel slope \\
\hline gap & $5 \times 10^{-4} \mathrm{~m}$ & Gap between coating and steel surface \\
\hline phi $_{0}$ & $-0.9 \mathrm{~V}$ & Measuring potential of body phase \\
\hline
\end{tabular}

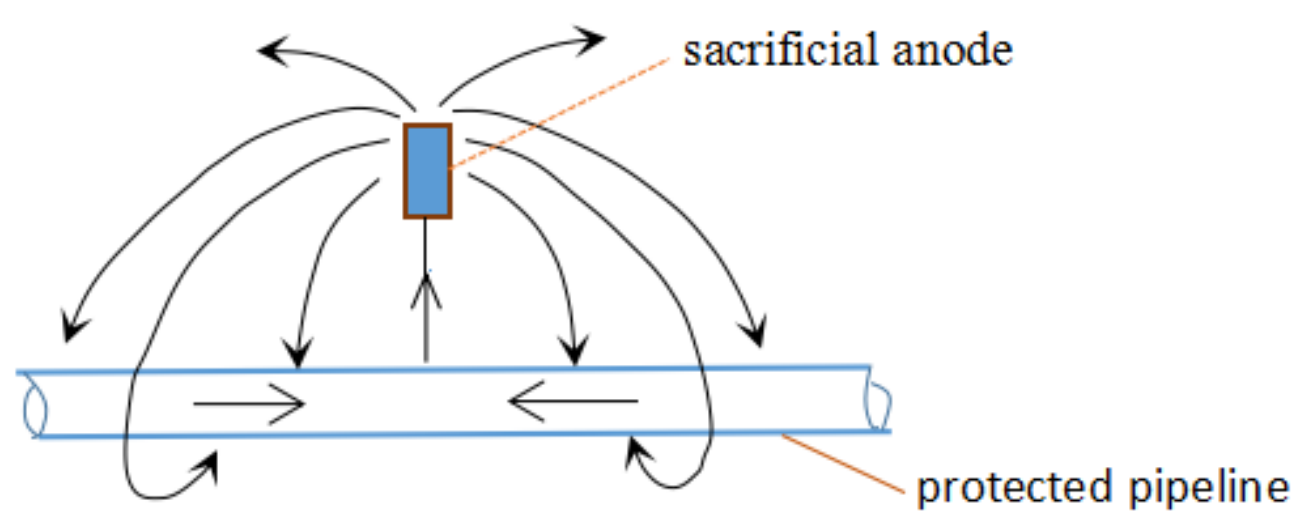

Fig. 2. Scheme of pipeline protection by sacrificial anode

\section{Results and discussions}

\section{Parallel pipelines}

Figure 3 shows the calculated potential distributions for parallel pipelines in a cathodic protection system, with a voltage of $10 \mathrm{~V}$ applied between the anode and the protected cathode, which is consistent with literature data [8,9] and actual project construction. Different colors represent potential magnitudes, where red and blue mean positive and negative values, respectively. As shown in Figure 3, the potential at the position of $0 \sim 20 \mathrm{~cm}$ is much higher than at other parts of the tank along the pipeline direction. Figure $3(b)$ shows the potential contour profile surrounding the parallel pipelines in detail. As shown in Figure 4, currents flow into the cathodically protected pipeline and return to the anode through the unprotected pipeline where corrosion occurs due to the dissolution reaction of the anode. Because there is potential gradient surrounding the anode, anode will interfere with the current flow path, that is, protected pipeline can accept current near the anode and then leading to current flowing along the pipeline. That is why the protected pipeline has more negative potential than the unprotected pipeline. 


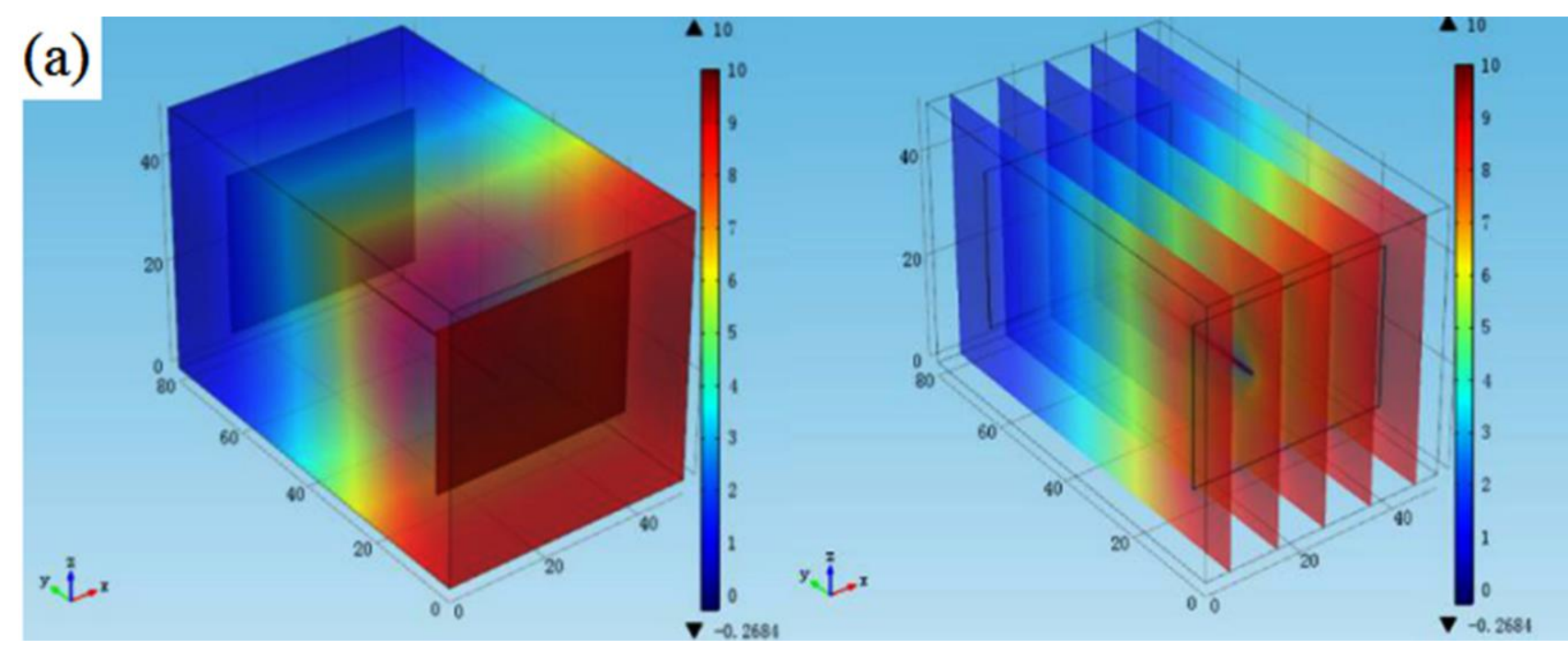

(b)

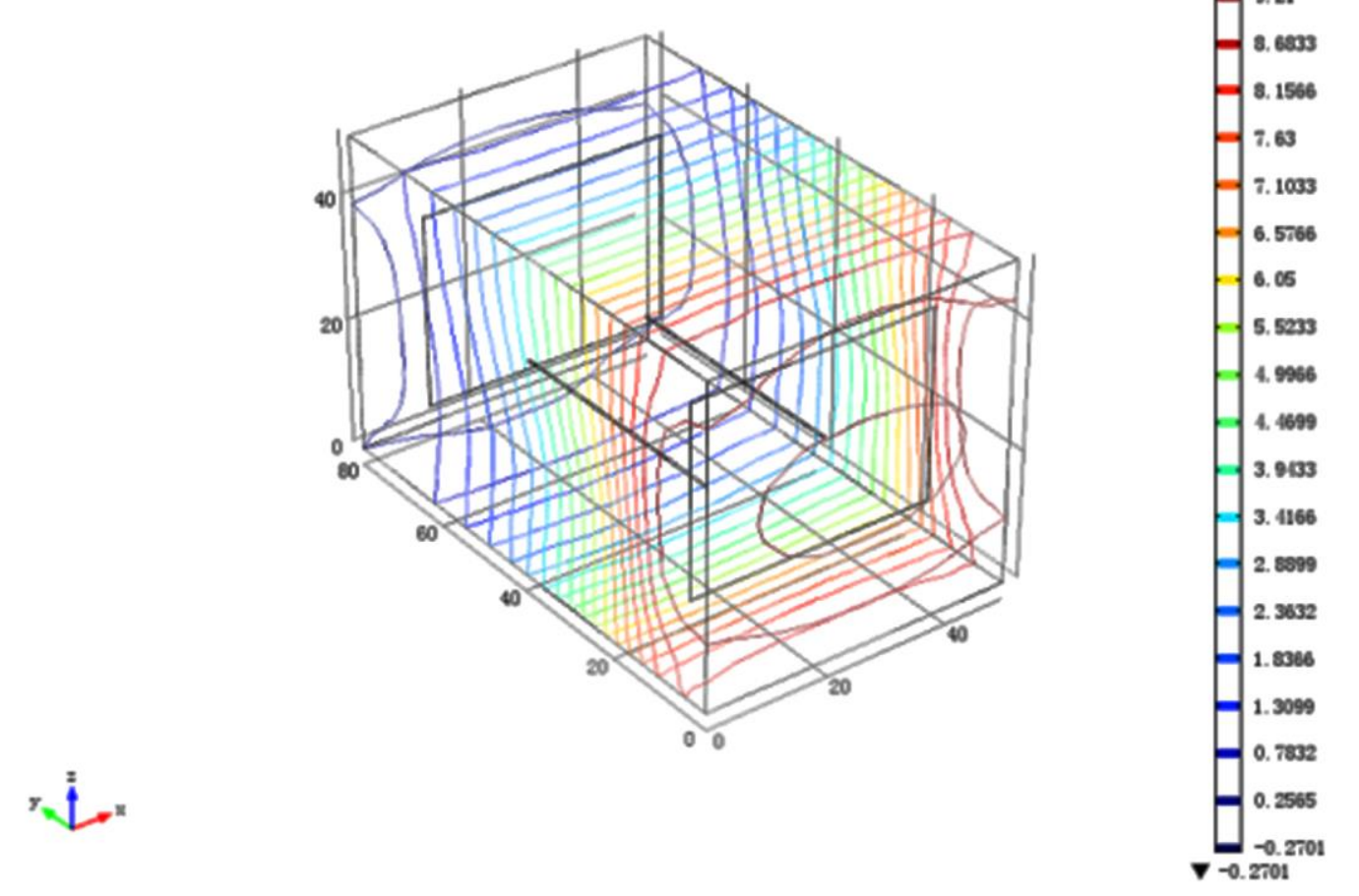

Fig. 3. Calculated potential distributions for parallel pipelines: (a) profile of equipotential surfaces; (b) chart of equipotential lines

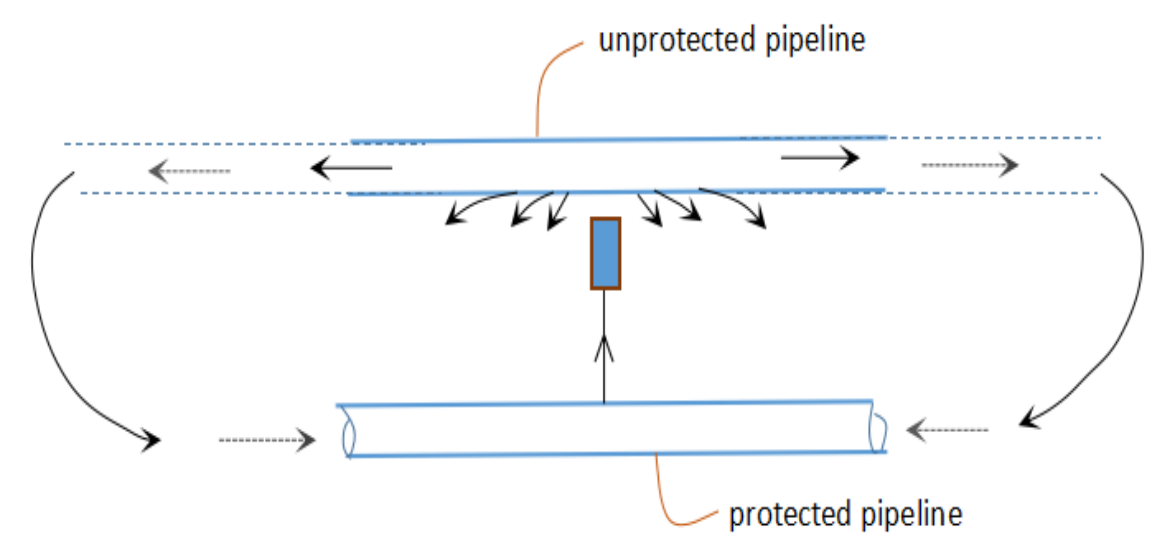

Fig. 4. Current flow direction for parallel pipelines 
a

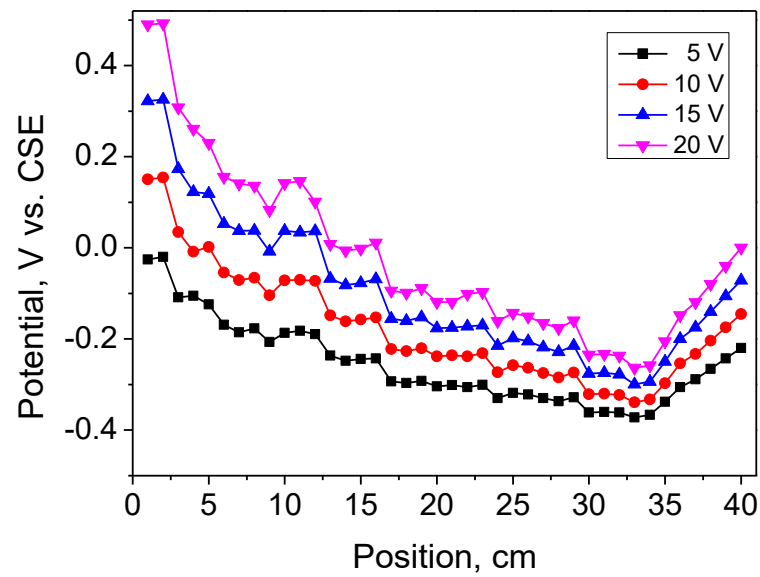

C



b

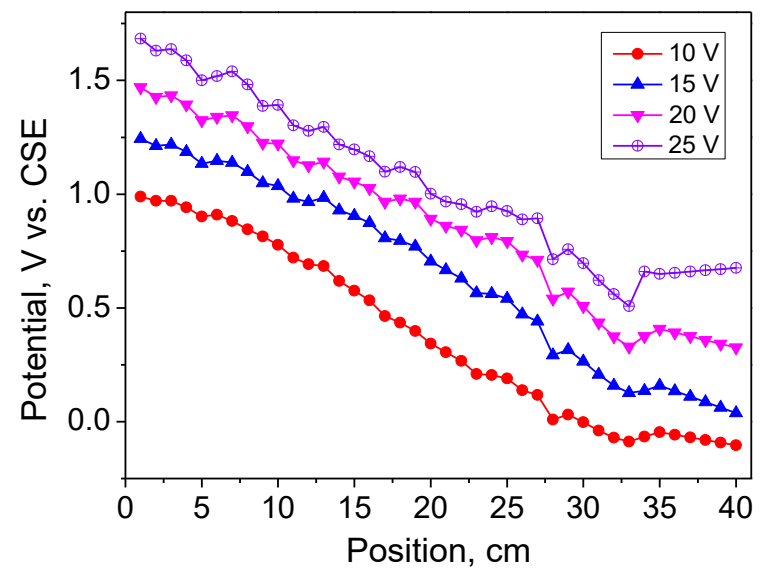

d

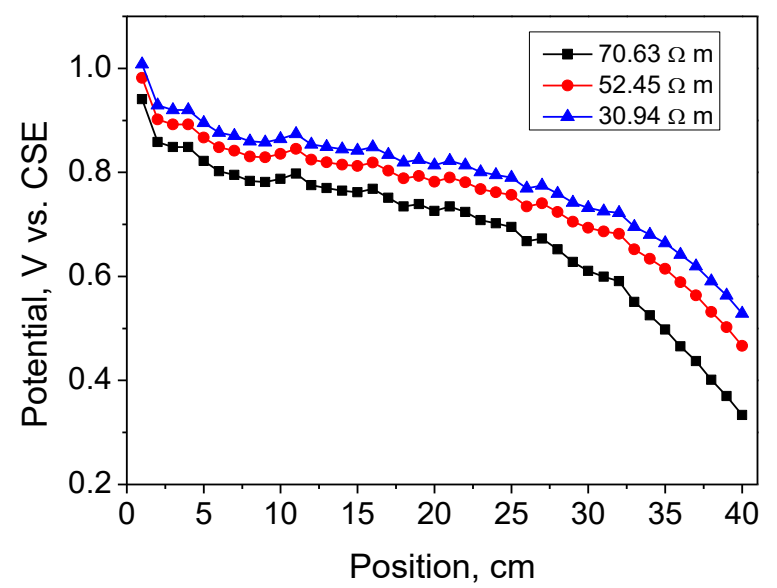

Fig. 5. Potential profiles along the parallel pipeline axial length: protected pipeline at (a) different voltage and (c) different soil resistivity; unprotected pipeline at

(b) different voltage and (d) different soil resistivity

Dependences of potential profiles on positions of protected/unprotected pipeline for different voltages are shown in Figure 5 . The potential of the unprotected pipeline is more positive than the protected pipeline, which suggests that the unprotected pipeline will be corroded firstly. At the voltage of $10 \mathrm{~V}$, the potential of the protected pipeline ranges from $-0.30 \mathrm{~V}$ to $0.17 \mathrm{~V}$. For the unprotected pipeline at $10 \mathrm{~V}$, however, the potential values are higher, and ranged between $0.00 \mathrm{~V}$ and $1.00 \mathrm{~V}$. According to the calculated data, it is proved that the sacrificial anode protection is effective. With voltage increase, the potentials show a positive trend of changes. According to Figure $5(\mathrm{a})$, at the position of $10 \mathrm{~cm}$, the potential rises from $-0.20 \mathrm{~V}$ to $0.20 \mathrm{~V}$ when the applied voltage increases from $5 \mathrm{~V}$ to $20 \mathrm{~V}$. Likewise, as shown in Figure 5(c) and (d), the potential profile shifts positively when the soil resistivity decreases, what can accelerate charge transfer and facilitate the process of corrosion.

The calculated potential distributions for crossed pipelines at a voltage of $10 \mathrm{~V}$ are shown in Figure 6 . The protected pipeline is situated along $y$-axis. It can be seen that the potential of the pipeline parallel to $y$-axis decreases progressively along the positive $y$-axis and the color changes from red to blue.

Potential versus position profiles for different voltages and soil resistivities of protected/unprotected pipelines are shown in Figure 7. For protected pipelines, the trend of potential change in Figures $7(\mathrm{a})$ and (c) is similar to Figures $5(\mathrm{a})$ and (c) and shows monotonous potential decrease. 


\section{Crossed pipelines}

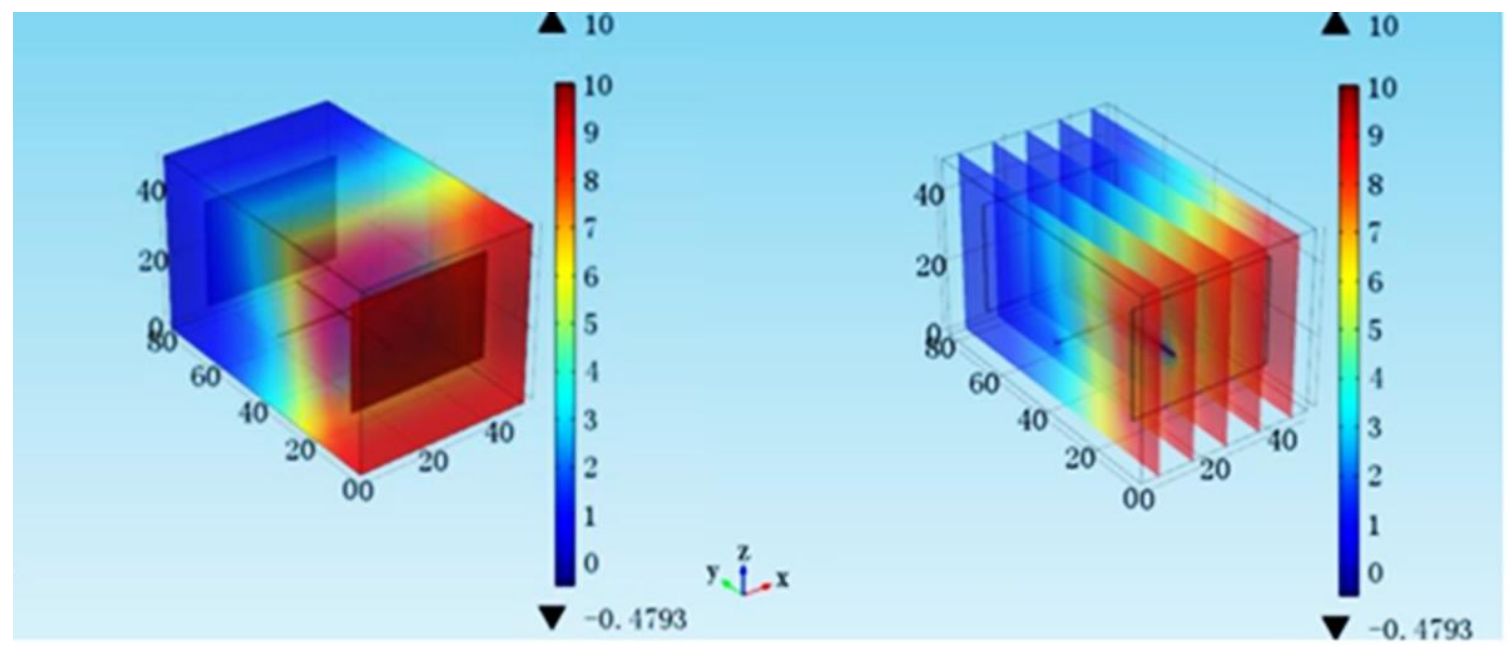

Fig. 6. Calculated potential distributions for crossed pipelines

a

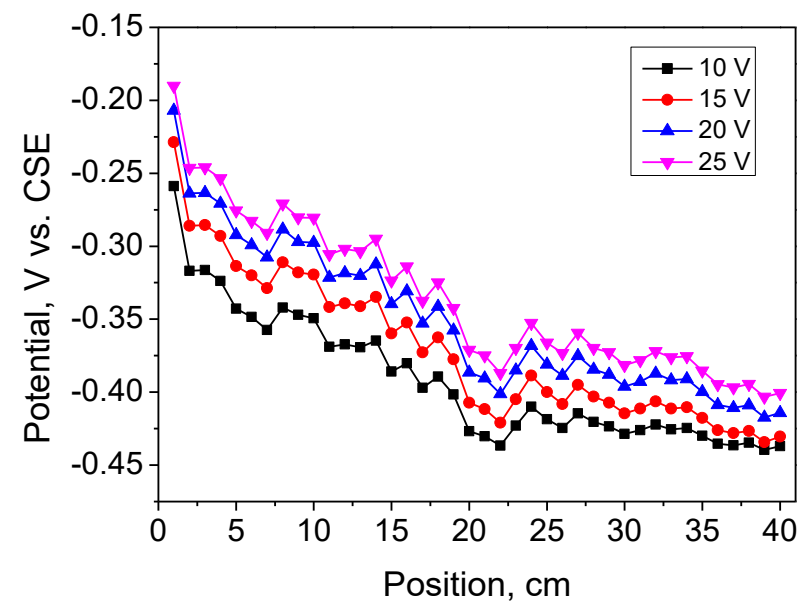

C

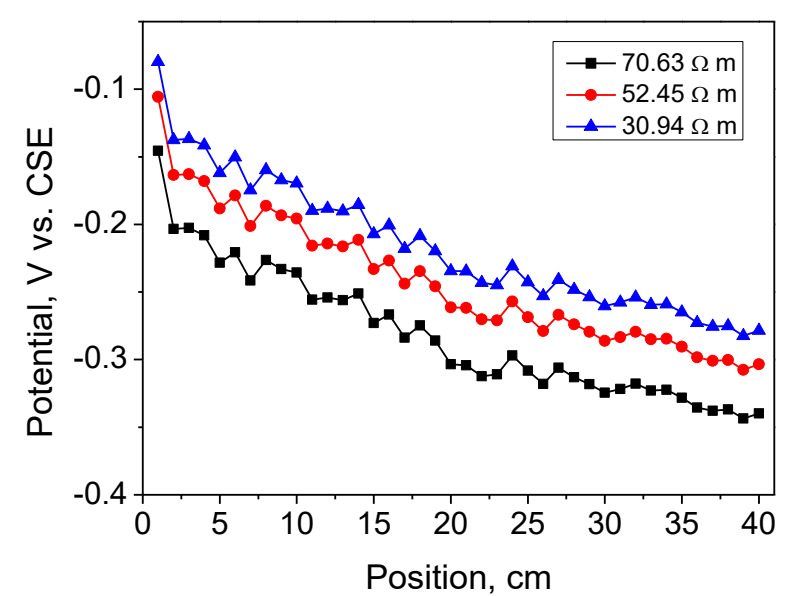

b

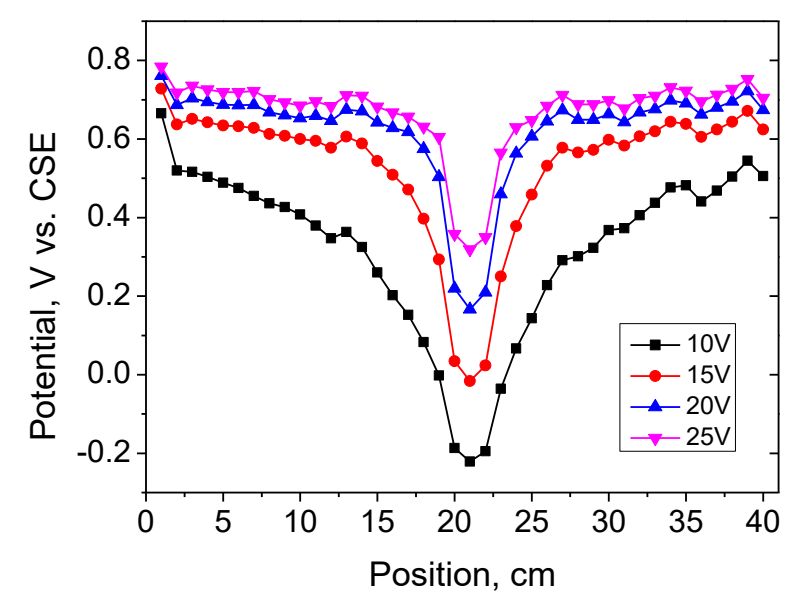

d

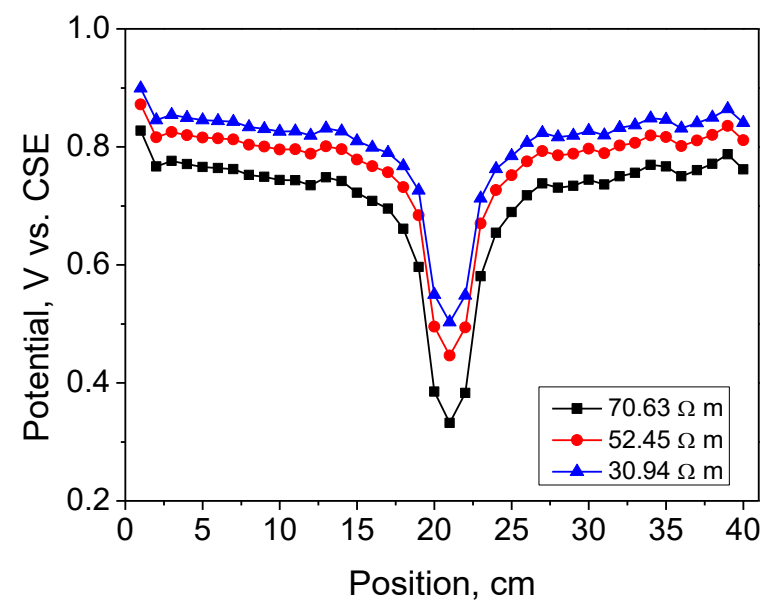

Fig. 7. Potential profiles along the crossed pipeline axial length: protected pipeline at (a) different voltage and (c) different soil resistivity; unprotected pipeline at (b) different voltage and (d) different soil resistivity

The stray current transfers from one broken side of protected pipeline to another broken side where corrosion occurs seriously. Potential distributions for unprotected pipelines shown in Figures 
7(b) and (d) are, however, significantly different from that shown in Figures 5(b) and (d). Both potential profiles exhibit a sharp downturn with the change of position, reaching a minimum value in the middle of pipeline and then begin to increase. Simply speaking, the potential distribution is presented as a letter " $\mathrm{V}$ ". In other words, the potential on the unprotected pipeline is symmetrical perpendicular to the protected pipeline. As shown in Figure 7(a) and (b), in the middle position at $20 \mathrm{~cm}$, the potential of the unprotected pipeline reaches $-0.20 \mathrm{~V}$, what is more positive than -0.42 $\mathrm{V}$ attained for the protected pipeline. Thus, the unprotected pipeline will suffer from severely concentrated corrosion. The corrosion reaches the maximum in the middle zone where the current flows out from the unprotected pipeline. As shown in the scheme drawn in Figure 8, the current flows out from the anode, enters the unprotected pipeline at the remote areas and then escapes from the crossed point of two pipelines. Since the potential of the unprotected pipeline is generally positive, a minimal potential value can be obtained at the position of current flowing out.

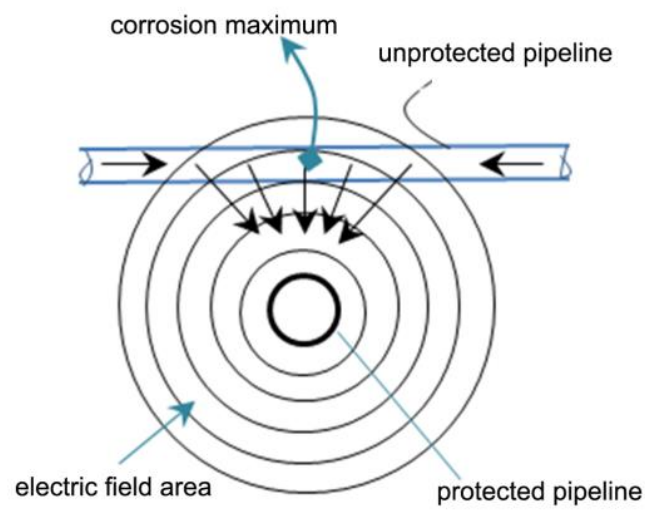

Fig. 8. Current direction for crossed pipelines

\section{Conclusions}

A mathematical model was developed to solve Laplace's three-dimensional equation with nonlinear boundary conditions for a cathodic protection system, and applied for considering the interference of DC stray current corrosion. Two different arrangements of protected and unprotected pipelines (parallel and crossed) were studied, and potential distributions of buried pipelines were for both arrangements obtained by simulations. It was shown that the type of protected/unprotected pipelines arrangements have a great impact on the potential distribution of the unprotected pipeline. The results also showed that both applied voltage and soil resistivity are crucial factors impacting stray current corrosion. Again, the sacrificial anode protection in a cathodic protection system was certified as an effective way for corrosion prevention.

Acknowledgements: The work described in this article was supported by grants from the National Natural Science Foundation of China (No.21476262), Technology Project of Qingdao 14-2-4-108-jch. The authors also acknowledge the associated support service by Associate Professor Xiao Tang's research group.

\section{References}

[1] L. Bertolini, M. Carsana, Corrosion science 49 (2007) 1056-1068.

[2] I. A. Metwally, H. M. Al-Mandhari, A. Gastli, Z. Nadir, Engineering Analysis with Boundary Elements 31 (2007) 485-493.

[3] A. I. H. Committee, S. D. Cramer, B. S. Covino. Stray-current corrosion in corrosion: Fundamentals, testing and protection, pp. 95-263, ASM International, 2003. 
[4] E. B. Muehlenkamp, M. D. Koretsky, J. C. Westall, Corrosion 61 (2005) 519-533.

[5] H. Al-Mandhary, I. A. Metwally, Z. Nadir, A. Gastli, A. A. Maqrashi, International Conference on Communication, Computer \& Power, 2007.

[6] I. A. Metwally, A. H. Al-Badi, Materials and Corrosion 61 (2010) 245-251.

[7] R. Strommen, W. Keim, J. Finnegan, et al, Materials Performance 26 (1987) 2-6.

[8] Y. Hong, L. Zuohua, Q. Guofu, Q. Jinping, Construction and Building Materials 157 (2017) 416-423.

[9] Y. S. Kim, G. J. Jeong and H. J. Sohn, Metals and Materials 5 (1999) 93-99.

[10] J. Warkus, M. Raupach, Materials and Corrosion 59 (2008) 122-130.

[11] I. A. Metwally, H. M. Al-Mandhari, Z. Nadir, A. Gastli, European Transactions on Electrical Power 17 (2007) 486-499.

[12] Md. A. Salam, Q. M. Rahman, S. P. Ang, F. Wen, Journal of Modern Power System and Clean Energy, 5 (2017) 290-297.

C)2019 by the authors; licensee IAPC, Zagreb, Croatia. This article is an open-access article distributed under the terms and conditions of the Creative Commons Attribution license (http://creativecommons. org/licenses/by/4.0/) 